\title{
Neuroimagen funcional y lenguaje
}

\author{
Joan Deus Yela
}

Unidad de Investigación en Resonancia Magnética. CRC-Hospital del Mar. Parque de Investigación Biomédica de Barcelona. Departamento de Psicología Clínica y de la Salud. Facultad de Psicología. Universitat Autònoma de Barcelona.

\section{Resumen}

El lenguaje es uno de nuestros dominios cognitivos más preciados. El estudio de dicha función neuropsicológica en las tres últimas décadas mediante técnicas de neuroimagen, y específicamente a través de la resonancia magnética funcional (RMf), ha proporcionado nuevos datos acerca del procesamiento cerebral del lenguaje y ha ampliado los clásicos ya conocidos. Básicamente se pueden definir cinco ámbitos de interés que se detallan en el presente artículo. El primero se centra en delimitar las áreas cerebrales que definen el circuito neural del lenguaje. El segundo intenta definir con mayor detalle la implicación de las diferentes áreas cerebrales en los distintos componentes lingüísticos, especialmente en el fonológico, el sintáctico y el semántico. El tercero se circunscribe al estudio de la reorganización funcional del lenguaje tras un daño cerebral. El cuarto se centra en la posibilidad del estudio no invasivo de la lateralización cerebral del lenguaje en la planificación prequirúrgica. Finalmente, el estudio de la etiopatogenia y la anormal actividad funcional lingüística de los trastornos del desarrollo del lenguaje oral y escrito para comprender su probable anormalidad neurobiológica. No obstante, se asume que la RMf presenta limitaciones en el estudio del procesamiento cerebral del lenguaje y precisa de la información obtenida mediante otras técnicas de neuroimagen y neurofisiológicas.

(Alzheimer. Real Invest Demenc. 2012;51:28-36)

Palabras clave: neuroimagen funcional, resonancia magnética funcional, circuito neural del lenguaje, lenguaje.

\section{Abstract}

Language is one of our most precious cognitive domains. The study of the neuropsychological function in the last three decades through neuroimaging techniques, specifically functional magnetic resonance imaging (fMRI), has provided new data on brain processing of language and expanded the classically known. Basically, we can define five focus areas detailed in this article. The first focused on defining the brain areas that define the neural circuitry of language. The second attempt to further define the involvement of different brain areas in different linguistic components, especially at the phonological, syntactic and semantic processing. The third is confined to the study of functional language reorganization after brain damage. The fourth focuses on the possibility of non-invasive study of cerebral lateralization of language in presurgical evaluation. Finally, the study of the pathogenesis and abnormal functional activity linguistic developmental disorders of speech and writing to understand their probable neurobiological abnormality. However, it is assumed that fMRI has limitations in the study of cerebral processing of language and precise information obtained by other techniques of neuroimaging and neurophysiological.

\section{(Alzheimer. Real Invest Demenc. 2012;51:28-36)}

Keywords: functional neuroimaging, functional MRI, neural circuitry of language, language. 
El lenguaje (del provenzal lenguatgea y éste del latín lingua) es un importante dominio cognitivo humano que no está aislado del resto de otras funciones cognitivas ${ }^{1}$. No existe una definición universalmente aceptada, si bien se asume que el lenguaje humano permite una intencionada comunicación oral (lenguaje de los sonidos) y escrita (lenguaje formal mediante signos) de los pensamientos entre los individuos. Es una de nuestras más preciadas y complejas habilidades cognitivas con un desarrollo innato (lenguaje oral), o biológicamente determinado, y aprendido (lenguaje escrito).

Ello ha motivado que haya sido una de las funciones neuropsicológicas más amplia y tempranamente estudiada entre los neuropsicólogos y neurólogos conductuales. La historia más reciente del procesamiento cerebral del lenguaje data de finales del siglo XVIII y se inicia con Franz Joseph Gall (1758-1828), a quien se le atribuye una de las más tempranas descripciones de un caso de trastorno del lenguaje adquirido y relacionado con una lesión frontal izquierda. En los siglos XIX y XX hubo aportaciones de importantes neuroanatomistas que relacionaban directamente el normal funcionamiento del lenguaje con estructuras cerebrales específicas ${ }^{2-5}$. Así, la neuroanatomía del lenguaje se circunscribió básicamente en regiones corticales perisilvianas del hemisferio izquierdo, implicando el área de Broca (área 44 de Brodmann —BA44-), el área de Wernicke (área 22 de Brodmann -BA22-), la circunvolución angular (área 39 de Brodmann —BA39-), la circunvolución supramarginal (área 40 de Brodmann -BA40-) y el fascículo arqueado (FA) que conecta bidireccionalmente el área BA44 y BA22 $2^{1,6}$. A raíz de tales descubrimientos y afirmaciones ha ido surgiendo un progresivo y creciente interés por la comprensión de las bases neurales del lenguaje ${ }^{6}$, posibilitando la formulación de diversos modelos neurolingüísticos para explicar el procesamiento cerebral del lenguaje oral y escrito.
El conocimiento acerca de las bases neurobiológicas del lenguaje oral y escrito se ha incrementado en las tres últimas décadas con las diversas técnicas de neuroimagen estructural y funcional ${ }^{6-8}$, y en especial las que posibilita la resonancia magnética. Esta última permite el estudio funcional, mediante la resonancia magnética funcional (RMf), en vivo de las habilidades cognitivas. La eclosión de los datos obtenidos ha posibilitado ratificar y complementar los resultados de los clásicos y tempranos estudios de la neuropsicología y neurología conductual efectuados mediante el procedimiento de relacionar la clínica con la lesión cerebral adquirida $^{6,9-10}$.

Específicamente, los resultados obtenidos con la RMf en el estudio de las bases neurales del lenguaje permiten definir, al menos, cinco ámbitos de interés. El primero se centra en la neuroanatomía del lenguaje, es decir, en delimitar las áreas cerebrales que definen el circuito neural del lenguaje. El segundo estudia la posibilidad de definir con mayor detalle la implicación y participación de las diferentes áreas cerebrales lingüísticas en los distintos componentes del lenguaje (tabla 1). Un tercer ámbito, y de creciente interés, queda circunscrito al estudio de la reorganización cerebral funcional del lenguaje tras una lesión cerebral adquirida que ocasiona una afasia. El cuarto ámbito de trabajo se centra en la posibilidad del estudio no invasivo de la lateralización cerebral del lenguaje en la planificación prequirúrgica. Finalmente se encuentra el estudio de la etiopatogenia y la anormal actividad funcional lingüística de los trastornos del desarrollo del lenguaje oral y escrito para comprender la probable anormalidad de su neurobiología.

En relación con el primer ámbito de interés de estudio, se han podido ampliar el número de regiones cerebrales que procesan el lenguaje. Se ha constatado que éstas no se limitan a las clásicas áreas lingüísticas perisilvianas del hemisferio izquierdo y que involucran un vasto circuito neural cortical. No obstante, se ratifica que el procesamiento fonológico-sintáctico-semántico implica, básicamente, un circuito parieto-témporo-frontal fuertemente lateralizado en el hemisferio izquierdo ${ }^{6}$.
Recibido para su publicación: 9 de enero de 2012.

Aceptado para su publicación: 30 de enero de 2012.

Correspondencia: J. Deus Yela

E-mail: Joan.Deus@uab.cat 


\begin{tabular}{|c|c|c|}
\hline Componentes del lenguaje & $\begin{array}{l}\text { Áreas cerebrales según } \\
\text { el modelo clásico }\end{array}$ & Áreas cerebrales según los datos de la RMf \\
\hline Lenguaje espontáneo & Área de Broca o BA44 & \\
\hline $\begin{array}{l}\text { Planificación } \\
\text { de la articulación }\end{array}$ & & $\begin{array}{l}\text { Ínsula anterior HI; AMS; putamen HI; CC anterior y cabeza } \\
\text { del núcleo caudado bilateralmente; BA44 porción ventral. }\end{array}$ \\
\hline Recuperación de palabras & & Circunvolución frontal media HI; BA44-45. \\
\hline $\begin{array}{l}\text { Monitorización } \\
\text { de producción oral }\end{array}$ & & CF superior; AMS; BA40. \\
\hline Comprensión oral & Área de Wernicke o BA22 & \\
\hline Análisis acústico-fonológico & & $\begin{array}{l}\text { Corteza auditiva primaria HI para el lenguaje, porción } \\
\text { rostral y caudal de la CT superior HI; planum temporale. }\end{array}$ \\
\hline Análisis semántico & & $\begin{array}{l}\text { Porción rostral y caudal de la CT media HI; circunvolución } \\
\text { angular HI; CC posterior/precuneus. }\end{array}$ \\
\hline Procesamiento sintáctico & & BA44-45 HI; planum temporale; BA40. \\
\hline Comprensión de la prosodia & & CT superior HD, CC rostral y dorsal HD. \\
\hline Repetición & Fascículo arqueado & $\begin{array}{l}\text { Fascículo arqueado, fascículo longitudinal superior, } \\
\text { fascículo uncinado. }\end{array}$ \\
\hline Denominación & Circunvolución temporal inferior & \\
\hline Denominación de objetos & & CT inferior HI en su porción más caudal. \\
\hline Denominación de acciones & & CF inferior HI rostral y caudalmente. \\
\hline Lenguaje escrito & Circunvolución angular o BA39 & \\
\hline Procesamiento fonológico & & CT media HI en su porción más caudal. \\
\hline Procesamiento semántico & & CP inferior HI en su porción más caudal. \\
\hline
\end{tabular}

Tabla 1. Áreas cerebrales implicadas en los diferentes componentes lingüisticos, según los principales datos de la resonancia magnética funcional (RMf), en comparación con el modelo clásico del circuito neural del lenguaje. AMS: área motora suplementaria; BA: área de Brodmann; CA: circunvolución angular; CC: circunvolución cingulada; CF: circunvolución frontal; CP: circunvolución parietal; CT: circunvolución temporal; HD: hemisferio derecho; HI: hemisferio izquierdo.

De forma más detallada, y de acuerdo con una organización lobular y por áreas funcionales cerebrales, se implican en el lenguaje las siguientes estructuras corticales del hemisferio izquierdo 6 ,7-10:

a) En el lóbulo occipital se involucran la corteza occipital ventral y dorsal (aproximadamente BA18/19) y la corteza occipito-temporal ventral y dorsal (aproximadamente BA37).

b) En el lóbulo temporal se incluyen la circunvolución de Heschl (aproximadamente BA41), situada en la circunvolución temporal superior; el surco temporal superior rostral-caudal; la circunvolución temporal superior (aproximadamente BA22); el planum temporale ubicado en la circunvolución temporal superior y caudalmente respecto a BA41 (apro- ximadamente BA42); el polo temporal situado en la circunvolución temporal superior y media (aproximadamente BA38); la circunvolución temporal media rostral y caudalmente (aproximadamente BA21); la circunvolución temporal inferior (aproximadamente BA20); la circunvolución angular (aproximadamente BA39), y la circunvolución temporal superior anterior del hemisferio derecho.

c) En el lóbulo parietal destaca la circunvolución supramarginal situada ventral y dorsalmente en dicho lóbulo (aproximadamente BA40).

d) En el lóbulo frontal se involucran la circunvolución precentral (aproximadamente BA6 rostralmente y BA4 caudalmente), el área motora suplementaria en su porción anterior (aproximadamente BA6 rostral-dorsal), la circunvolución frontal superior 
(aproximadamente BA8), el surco frontal inferior (aproximadamente BA9), la circunvolución frontal media (aproximadamente BA46), la parte orbitalis de la circunvolución frontal inferior (aproximadamente BA47), la parte triangularis de la circunvolución frontal inferior (aproximadamente BA45), la parte opercular ventral y dorsal (aproximadamente BA44) y la corteza prefrontal dorsolateral derecha.

Obviamente, dichas estructuras corticales lingüísticas están funcional y estructuralmente conectadas de forma bidireccional. De forma específica se han definido dos conexiones dorsales (superiores) y dos ventrales (inferiores) $)^{6}$ :

a) Los fascículos dorsales, en primer término, permiten conectar la circunvolución temporal caudaldorsal con la corteza premotora (aproximadamente BA6) vía el FA y el fascículo longitudinal superior (FLS); el segundo fascículo dorsal conecta la circunvolución temporal superior, más rostralmente, con BA44 vía el FA y el FLS.

2. Los fascículos ventrales, en primer lugar, permiten conectar BA45 y la circunvolución temporal superior en su porción rostral vía la fibra extrema del sistema de cápsula; el segundo fascículo ventral conecta el opérculo frontal (aproximadamente BA38 ventralmente) y la circunvolución temporal superior en su porción rostral vía el fascículo uncinado.

Korbinian Brodmann fue el primer neuroanatomista que proporcionó una detallada descripción de la citoarquitectura de la corteza cerebral humana ${ }^{11}$. Sin embargo, el uso de la RMf y, sobre todo, el estudio de la parcelación de la conectividad funcional y estructural $^{12-15}$ ha permitido obtener una información fundamental para poder detallar con una mayor especificidad la nueva neuroarquitectura de ciertas regiones corticales implicadas en los componentes del lenguaje $\mathrm{e}^{6-10}$ (tabla 1). Posiblemente, en esta nueva línea de trabajo se ha hecho mayor hincapié en ciertas funciones lingüísticas tales como el procesamiento fonológico, el semántico y el lingüístico de textos y oraciones ${ }^{10}$.
En líneas generales, y a modo de resumen, parece ser que la circunvolución frontal inferior izquierda está especializada en el procesamiento fonológico y semántico, mientras que la parte orbitalis de la circunvolución frontal inferior está dedicada al procesamiento sintáctico y la circunvolución temporal superior se implica en la comprensión de oraciones y textos ${ }^{6-10}$. La capacidad de repetición, con y sin sentido semántico, puede depender de una activación bilateral ${ }^{16}$ o principalmente izquierda del FA ${ }^{17}$, que, a su vez, es estructuralmente asimétrico entre ambos hemisferios con un mayor grosor en el hemisferio izquierdo, tanto para diestros como para zurdos, y con un grosor similar para ambos hemisferios en los ambidiestros ${ }^{17}$. Sin embargo, el FA muestra una mayor conexión estructural con el BA47 de ambos hemisferios ${ }^{15}$. En relación con la capacidad de denominación, se ha demostrado un clásico postulado de un posible circuito neural diferente para la denominación de acciones y de objetos. Así, se ha constatado que la denominación de acciones por confrontación visual depende, principalmente, de un circuito neural lateralizado al hemisferio izquierdo a nivel de la circunvolución frontal inferior rostral y caudalmente. Por el contrario, en la denominación de objetos por confrontación visual se implica, principalmente, la circunvolución temporal inferior izquierda en su porción más caudal ${ }^{18}$. En relación con la comprensión del lenguaje escrito (lectura), finalmente, se han propuesto dos circuitos neurales: uno precisa de un procesamiento fonológico (ortografía no transparente) dependiente de la circunvolución temporal izquierda en su porción más caudal y otro necesita un procesamiento semántico (ortografía transparente) más dependiente de la circunvolución parietal inferior izquierda en su porción más caudal ${ }^{19}$.

Algunos autores matizan que el mayor desarrollo de las técnicas de RMf con mejor resolución tempo$\mathrm{ral}^{20}$ va a permitir en un futuro que dicha técnica pueda a llegar a ser más importante de lo que es ahora en el estudio del lenguaje ${ }^{8}$. Actualmente, por consiguiente, la RMf presenta limitaciones en el estudio del procesamiento cerebral del lenguaje y precisa de la información obtenida mediante otras técnicas de neuroimagen y neurofisiológicas ${ }^{8,14}$. A modo de ejemplo y 
en relación con la producción oral clásica, específicamente el BA44 se ha implicado de forma exclusiva en la fluencia de la expresión oral. No obstante, la producción de palabras, la gramática y la producción fonológica son distintas funciones lingüísticas que parecen ser dependientes de BA446,8-10 y que pueden parcelarse anatómicamente de acuerdo con un parámetro de actividad funcional temporal estudiado mediante la estimulación electrofisiológica intracraneal ${ }^{21}$. Así, Sahin y cols. (2009) muestran que la función léxica involucra una región de BA44 que implica una actividad neural de $200 \mathrm{~ms}$, la gramatical implica una actividad neural de BA44 de 320 ms y la fonológica, una actividad neural de BA44 de 450 ms. Estos resultados sugieren que BA44 computa diferentes pasos lingüísticos relacionados que no pueden detectarse exclusivamente con la RMf.

En relación con el estudio de la reorganización cerebral funcional del lenguaje tras una lesión cerebral adquirida, la RMf proporciona datos de gran interés neurocientífico. Brevemente, la afasia se define como la pérdida parcial o completa del lenguaje oral y/o escrito, previamente adquirido, de forma secundaria a una lesión cerebral adquirida en áreas cerebrales de asociación unimodal implicadas en el lenguaje. En las pasadas décadas se ha podido constatar un importante avance en el diagnóstico y el tratamiento de la afasia, con poco conocimiento acerca de los cambios neurales que se implican y promueven la recuperación funcional del lenguaje ${ }^{22}$. Sin embargo, recientes estudios de RMf están contribuyendo a la comprensión de la pérdida y recuperación funcional, en los estadios agudos y crónicos, de las habilidades lingüísticas tras un daño cerebral focal adquirido ${ }^{23}$. Brevemente, los estudios de RMf sugieren dos importantes datos. En primer lugar, que la presencia de focos de activación funcional en el hemisferio derecho no lesionado, en la ejecución de una tarea lingüística, durante la fase aguda o en el momento basal del inicio de la rehabilitación logopédica o biológica (farmacoterapia o estimulación magnética transcraneal - TMS-), puede relacionarse con una buena recuperación de la afasia a largo plazo. En segundo lugar, que una persistencia temporal de la activación funcional de la corteza fron- tal derecha durante la ejecución en tareas de lenguaje en estadios evolucionados de una afasia impide una mayor recuperación de los déficits lingüísticos. Ello pueda explicarse, probablemente, al dificultar que las áreas lingüísticas del hemisferio izquierdo preservadas puedan implicarse en la reorganización funcional del lenguaje perdido ${ }^{22-24}$.

El estudio no invasivo de la lateralización cerebral del lenguaje en la planificación prequirúrgica ha supuesto la aplicabilidad clínica de la RMf ${ }^{25}$, con una sensibilidad del $83,5 \%$ y una especificidad del $88,1 \%$ respecto al clásico test de Wada ${ }^{26}$ (fig. 1) Para ello se han propuesto varias tareas lingüísticas, y se ha llegado a la conclusión de que la fluencia verbal con consigna fonética y la generación de verbos son las más idóneas para dicho propósito. Así, en un ya clásico y ratificado estudio de RMf, se ha constatado que los diestros muestran un lenguaje lateralizado en el hemisferio izquierdo en el $96 \%$ de los casos, mientras que los zurdos tienen representado el lenguaje en el hemisferio izquierdo en un $76 \%$, en el derecho en un $10 \%$ y bilateralmente el $14 \%$ restante $^{27}$ (fig. 2). No obstante, los resultados obtenidos con RMf en pacientes prequirúrgicos siempre han de ser interpretados con precaución al evaluarse una compleja función cognitiva y por la presencia de posibles artefactos vasculares ${ }^{25}$. Por ello, la localización de áreas cerebrales lingüísticas elocuentes en pacientes prequirúrgicos no es tan extensamente aplicable al ámbito clínico. Sin embargo, en determinadas tareas de lenguaje se han podido delimitar específicas regiones corticales lingüísticas, tales como la BA22 ${ }^{28}$, la BA39 o la dimensión lingüística del área motora suplementaria (fig. 3).

Finalmente, la RMf permite el estudio de las posibles anormalidades en la lateralización cerebral del lenguaje en determinadas patologías neurológicas, tales como los pacientes epilépticos o con trastornos del desarrollo del lenguaje oral y escrito. En los primeros se asume una alta probabilidad de una atípica organización cerebral para el lenguaje. Existen suficientes datos que sugieren que la naturaleza crónica de la actividad epileptiforme, y de otras variables clínicas, pueden producir dos fenómenos: el primero, ocasionar un cambio en el habitual neurodesarrollo de la laterali- 


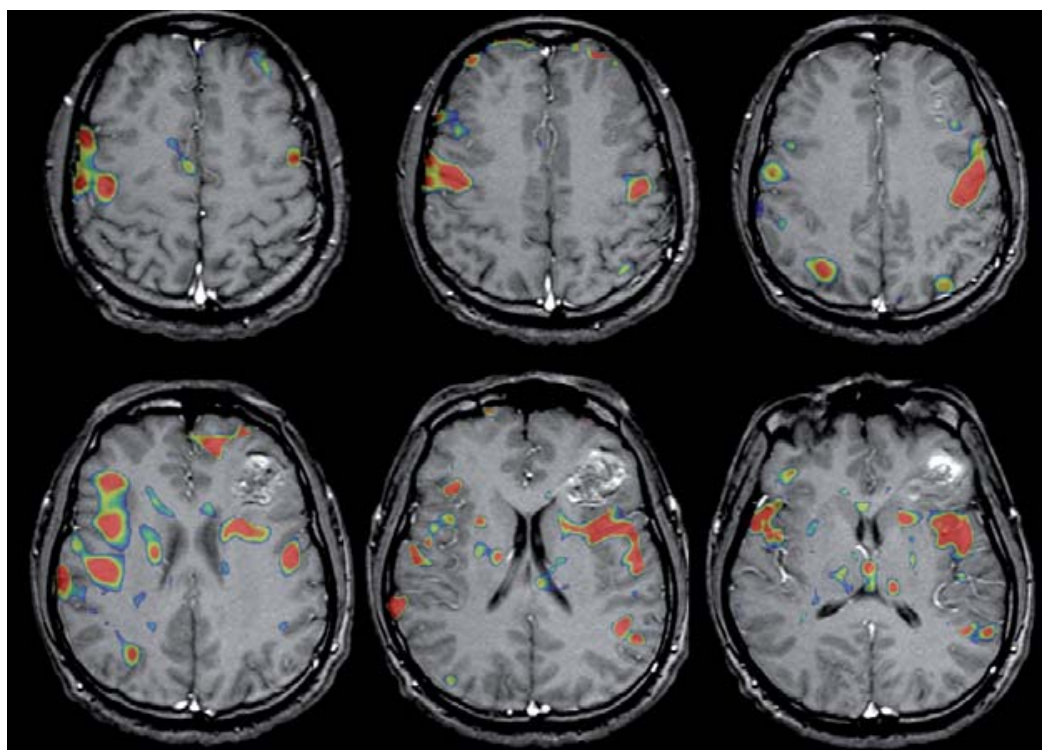

Figura 1. Paciente manidiestro con una lesión ocupante del espacio prefrontal izquierdo. La RMf del lenguaje muestra un patrón de activación funcional significativa de la corteza sensitivo-motora bilateral, premotora bilateral, de la cabeza y cuerpo del núcleo caudado bilateral de predominio izquierdo, prefrontal dorsolateral de predominio derecho, de la neocorteza prefrontal dorsolateral más anterior izquierda, de la circunvolución occipito-parietal derecha e insular bilateral. La RMf de lenguaje sugiere una participación de ambos hemisferios en tareas de fluencia verbal con consigna fonética.

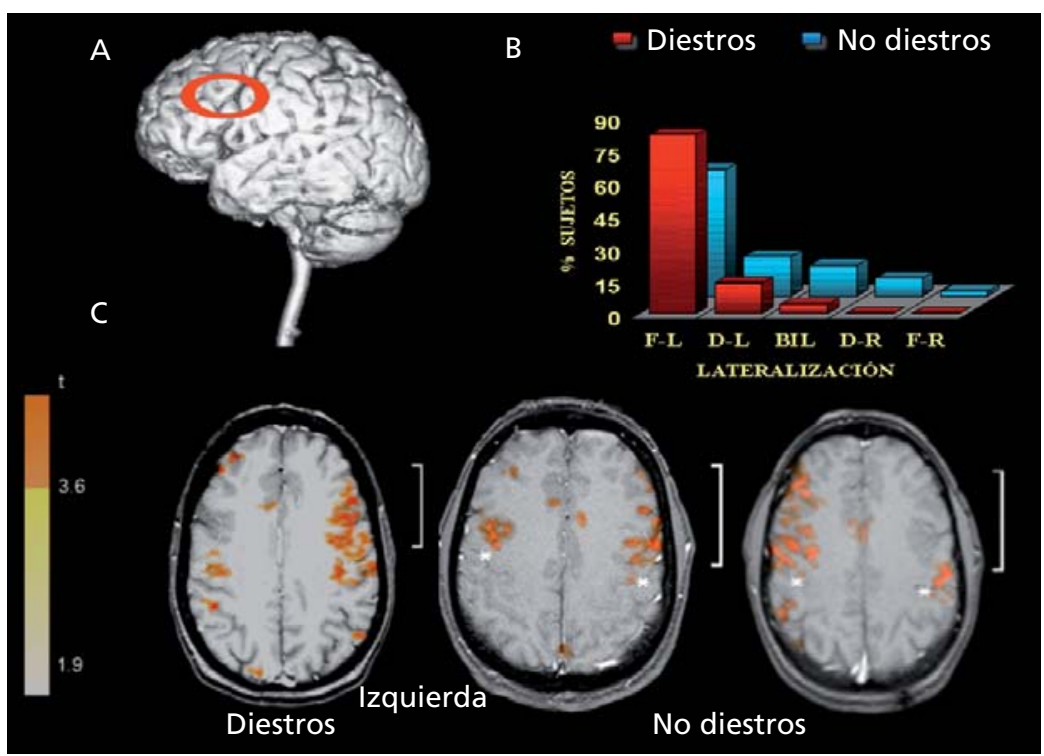

Figura 2. A: Corte sagital en el que se señala el surco frontal inferior (aproximadamente BA9) y la circunvolución frontal media (aproximadamente BA46) como áreas implicadas en la fluencia verbal con consigna fonética. B: Porcentaje de lateralización cerebral para el lenguaje en sujetos diestros y no diestros (F-L: fuertemente hemisferio izquierdo; D-L: débilmente hemisferio izquierdo; BIL: Bilateral; D-R: débilmente hemisferio derecho; F-R: fuertemente hemisferio derecho). C: Cortes axiales de RMf que muestran los tres posibles patrones de activación funcional cerebral, en tarea de fluencia verbal con consigna fonética, en sujetos diestros y no diestros. 


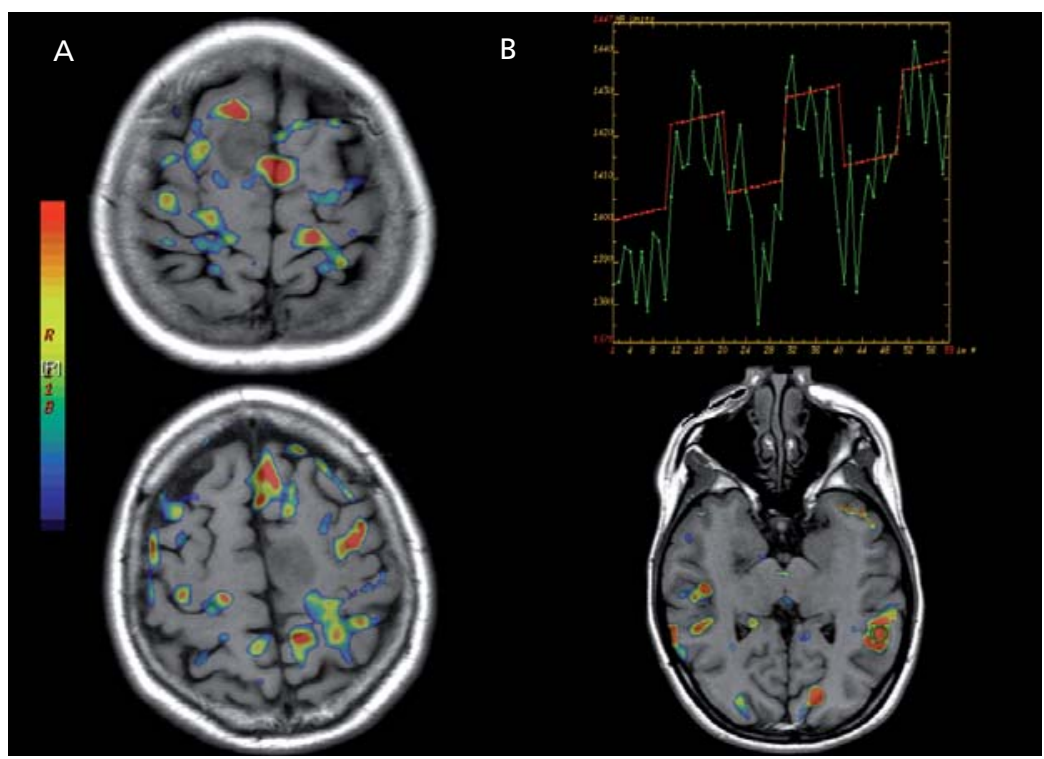

Figura 3. A: Cortes axiales de RMf que muestran el patrón de activación funcional de la porción anterior (dimensión lingüística) del área motora suplementaria mediante la tarea verbal de generación de verbos. B: La figura superior muestra el patrón de ejecución realizada por el sujeto en estudio (línea verde), durante la adquisición de la RMf y mientras realiza la tarea lingüística de lectura, y el patrón de ejecución ideal (línea roja). En la figura inferior, corte axial de RMf durante la tarea de lectura atenta y comprensiva en voz silente en la que se constata la activación funcional de la circunvolución angular (BA39) del hemisferio izquierdo.

zación del lenguaje en el hemisferio izquierdo al derecho; el segundo, originar una transformación de los tradicionales circuitos neurales lingüísticos del hemisferio izquierdo a otros no tradicionales y situados en el mismo hemisferio ${ }^{29}$.

En los trastornos del desarrollo del lenguaje oral y escrito se ha postulado que la anormal lateralización funcional del lenguaje puede ser una de sus posibles causas etiológicas o de su anormal curso evolutivo. Se ha constatado que en un $32,2 \%$, específicamente en sujetos manidiestros, se objetiva una anormal lateralización cortical del lenguaje con un patrón de representación bilateral ${ }^{30}$. Ello podría explicar y conllevar un déficit en el procesamiento fonológico, dependiente de un circuito neural frontoparietal izquierdo, en niños con dislexia del desarrollo ${ }^{31}$. Finalmente, el normal neurodesarrollo de las habilidades lingüísticas depende de una correcta neuromaduración de la sustancia blanca y, específicamente, en las áreas lingüísticas perisilvianas. Ello posibilitará una buena lateralización funcional del lenguaje y un buen desarrollo funcional de dicha función cognitiva. Así, el rápido y eficaz desarrollo de la riqueza de la expresión oral de un niño coincide con una aceleración progresiva de la génesis de sustancia blanca perisilviana del hemisferio izquierdo ${ }^{32}$.

\section{Agradecimientos}

El Dr. Joan Deus pertenece al Grupo de Investigación SGR 1450 de l'Agència de Gestió d'Ajuts Universitaris i de Recerca de la Generalitat de Catalunya y la elaboración del presente artículo ha sido en parte apoyado por Plan Nacional de I+D+I (Beca SAF2007-62376) del Ministerio de Educación y Ciencia de España.

\section{Conflictos de interés}

El autor declara la ausencia de conflictos de interés. 


\section{Bibliografía}

1. Berthier ML, Green C. Afasia: formas clinicotopográficas y modelos funcionales. En: Peña-Casanova J, ed. Neurología de la conducta y neuropsicología. Madrid: Editorial Médica Panamericana; 2007. p. 93-107.

2. Broca P. Remarques sur le siége de la faculté du langage articulé, suivies d'une observation d'aphémie (parte de la parole). Bull Soc Anat Paris. 1861;6:330-57.

3. Lichtheim L. On aphasia. Brain. 1884;7:433-84.

4. Wernicke C. Der aphasische Symptomencomplex. Berlín: Springer-Verlag; 1874.

5. Bassetti CL, Jagella EC. Joseph Jules Dejerine (18491917). J Neurol. 2006;253:823-4.

6. Friederici AD. The brain basis of language processing: from structure to function. Physiol Rev. 2011;91:1357-92.

7. Binder JR. Where is the semantic system? A critical review and meta-analysis of 120 functional neuroimaging studies. Cereb Cortex. 2009;19:2767-96.

8. Cunillera-Llorente T, Rodriguez-Fornells A. Lenguaje I: Expresión y comprensión. En: Maestú-Unturbe F, RíosLago M, Cabestrero-Alonso R, eds. Neuroimagen. Técnicas y procesos cognitivos. Ámsterdam: Elsevier Masson; 2008. p. 413-32.

9. Price CJ. The anatomy of language: a review of $100 \mathrm{fMRI}$ studies published in 2009. Ann NY Acad Sci. 2010:1191: 62-88.

10. Vigneau M, Beaucousin V, Hervé PY, Duffau H, Crivello F, Houdé $\mathrm{O}$, et al. Meta-analyzing left hemisphere language areas: phonology, semantics, and sentence processing. Neuroimage. 2006;30:1414-32.

11. Brodmann K. Beiträge zur histologischen Lokalisation der Grosshirnrinde. VI. Die Cortexgliederung des Menschen. J Psychol Neurol. 1909;10:231-46.

12. Anwander A, Tittgemeyer M, Von Cramon DY, Friederici AD, Knösche TR. Connectivity-based parcellation of Broca's area. Cereb Cortex. 2007;17:816-25.

13. Johansen-Berg H, Behrens TEJ, Robson MD, Drobnjak I, Rushworth MFS, Brady JM, et al. Changes in connectivity profiles define functionally distinct regions in human medial frontal cortex. Proc Natl Acad Sci. 2004;1001: 13335-40.

14. Ford A, McGregor KM, Case K, Crosson B, White KD. Structural connectivity of Broca's area and medial frontal cortex. Neuroimage. 2010;52:1230-37.

15. Kaplan E, Naeser MA, Martin PI, Ho M, Wang Y, Baker E, et al. Horizontal portion of arcuate fasciculus fibers track to pars opercularis, not pars triangularis, in right and left hemispheres: A DTI study. Neuroimage. 2010;52: 436-44.
16. Brendel B, Hertrich I, Erb M, Lindner A, Riecker A, Grodd W, et al. The contribution of mesiofrontal cortex to the preparation and execution of repetitive syllable productions: An fMRI study. Neuroimage. 2010;50:1219-30.

17. Propper RE, O'Donnell LJ, Whalen S, Tie Y, Norton IH, Suarez RO, et al. A combined fMRI and DTI examination of functional language lateralization and arcuate fasciculus structure: Effects of degree versus direction of hand preferente. Brain Cogn. 2010:73:85-92.

18. Liljeström M, Tarkiainen A, Parviainen T, Kujala J, Numminen J, Hiltunen J, et al. Perceiving and naming actions and objects. Neuroimage. 2008;41:1132-41.

19. Das T, Padakannaya P, Pugh KR, Singh NC. Neuroimaging reveals dual routes to reading in simultaneous proficient readers of two orthographies. Neuroimage. 2011; 54:1476-87.

20. Le Bihan D. Looking into the functional architecture of the brain with diffussion MRI. Nat Rev Neurosci. 2003; 4:469-80.

21. Sahin NT, Pinker S, Cash SS, Schomer D, Halgren E. Sequential processing of lexical, grammatical, and phonological information within Broca's area. Science. 2009; 326:445-9.

22. Berthier ML, García-Casares N, Walsh SF, Nabrozidis A, Ruiz de Mier RJ, Green C, et al. Recovery from post-stroke aphasia: lessons from brain imaging and implications for rehabilitation and biological treatments. Discov Med. $2011 ; 12: 275-89$.

23. Saur D, Hartwigsen G. Neurobiology of language recovery alter stroke: lessons from neuroimaging studies. Arch Phys Med Rehabil. 2012;93:S15-25.

24. Turkeltaub PE, Coslett B, Thomas AL, Faseyitan O, Benson J, Norise C, et al. The right hemisphere is not unitary in its role in aphasia recovery. Cortex. , 2011;Jun 30. [Epub ahead of print].

25. Peck KK, Bradbury M, Petrovich N, Hou BL, Ishill N, Brennan $\mathrm{C}$, et al. Presurgical evaluation of language using functional magnetic resonance imaging in brain tumor patients with previous surgery. Neurosurgery. 2009;64: 644-53.

26. Dym RJ, Burns J, Freeman K, Lipton ML. Is functional MR Imaging assessment of hemispheric language dominante as good as the Wada test?: a meta-analysis. Radiology. 2011;26:446-55.

27. Pujol J, Deus J, Losilla JM, Capdevila A. Cerebral lateralization of language in normal left-handed people studied by functional MRI. Neurology. 1999:52:1038-43.

28. Sanjuán A, Forn C, Ventura-Campos N, RodríguezPujadas A, García-Porcar M, Belloch V, et al. The sentence verification task: a reliable fMRI protocol for mapping 
receptive language in individual subjects. Euro Radiol. 2010;20:2432-8.

29. Hamberger MJ, Cole J. Language organization and reorganization in epilepsy. Neuropsychol Rev. 2011;21:24051.

30. Sans A, Pujol J, Deus J, Boix C, López-Sala A, Colomé R. Resonancia magnética funcional: su utilidad en neuropsicología infantil. Rev Neurol Clin. 2001;2:72-85.
31. Wolf RC, Sambataro F, Lohr C, Steinbrink C, Martin C, Vasic N. Functional brain network abnormalities during verbal working memory performance in adolescents and young adults with dyslexia. Neuropsychologia. 2010;48: 309-18.

32. Pujol J, Soriano-Mas C, Ortiz H, Sebastián-Gallés N, Losilla JM, Deus J. Myelination of language-related areas in the developing brain. Neurology. 2006;66:339-43. 